PAPER

\title{
Auditory disturbance as a prodrome of anterior inferior cerebellar artery infarction
}

\section{H Lee, Y-W Cho}

See end of article for authors' affiliations

....................

Correspondence to:

Dr Hyung Lee, Department

of Neurology, School of

Medicine, Keimyung

University, 194 Dongsan

dong, Daegu 700-712

South Korea;

hlee@dsmc.or.kr

Received

19 December 2002

In revised form

28 April 2003

Accepted 29 April 2003

\begin{abstract}
Objectives: To investigate the clinical and radiological features of patients presenting with an acute auditory syndrome as a prodromal symptom of anterior inferior cerebellar artery (AICA) infarction. Methods: 16 consecutive cases of AICA infarction diagnosed by brain magnetic resonance imaging completed a standardised audiovestibular questionnaire and underwent a neuro-otological evaluation by an experienced neuro-otologist.

Results: Five patients (31\%) had an acute auditory syndrome as a prodrome of AICA infarction one to 10 days before onset of other brain stem or cerebellar symptoms. Two types of acute auditory syndrome were found: recurrent transient hearing loss with or without tinnitus $(n=3)$, and a single episode of prolonged hearing loss with or without tinnitus $(n=2)$. The episodic symptoms were brief, lasting only minutes. The tinnitus preceding the infarction was identical to the tinnitus experienced at the time of infarction. At the time of infarction, all patients developed hearing loss, tinnitus, vertigo, and ipsilateral hemiataxia. The most commonly affected site was the middle cerebellar peduncle $(n=5)$. Four of the five patients had incomplete hearing loss and all had absence of vestibular function to caloric stimulation on the affected side.

Conclusions: Acute auditory syndrome may be a warning sign of impending pontocerebellar infarction in the distribution of the AICA. The acute auditory syndrome preceding an AICA infarct may result from ischaemia of the inner ear or the vestibulocochlear nerve.
\end{abstract}

A n acute auditory symptom without associated neurological symptoms or signs usually results from dysfunction of the peripheral auditory system located between the cochlea and the auditory nerve distal to the root of entry. ${ }^{1}$ The anterior inferior cerebellar artery (AICA) supplies the dorsolateral pons, middle cerebellar peduncle, inner ear, vestibulocochlear nerve, and anterior inferior cerebellum including the flocculus. ${ }^{1-5}$ Characteristically, an acute auditory symptom from vertebrobasilar insufficiency is associated with multiple brain stem or cerebellar signs. ${ }^{67}$ However, as the blood supply to the peripheral auditory system arises from the internal auditory artery, ordinarily a branch of the AICA, partial ischaemia in the AICA territory could lead to an isolated acute auditory syndrome such as hearing loss and tinnitus.

Recently, we reported that sudden hearing loss is a common sign of an AICA infarct. ${ }^{8}$ To the best of our knowledge, an acute auditory syndrome as a warning sign of impending pontocerebellar infarction has not been reported in large consecutive clinical series. Our aim in the present study was to investigate the frequency, characteristic features, and origin of the acute auditory syndrome as a prodromal feature of AICA infarction.

\section{METHODS}

Over 34 months (1 January 2000 to 30 October 2002), we prospectively identified 16 consecutive cases of a unilateral AICA infarction diagnosed by brain magnetic resonance imaging (MRI). In all cases a full history was taken and a neurological examination done by an experienced neurootologist. In addition, each patient completed a standardised audiovestibular questionnaire including a detailed description about the acute auditory syndrome. All patients underwent standard audiometric assessments including pure tone hearing levels to air and bone conduction, tympanometry, an acoustic impedance study, and auditory brain stem responses
(ABR). Quantitative electronystagmography was also undertaken on each patient to document the vestibular deficits. Detailed descriptions of the audiovestibular tests have been published previously. ${ }^{8}$

All neuro-otological evaluations were done during the acute phase (one to four days) after the AICA infarct. MRI and magnetic resonance angiography (MRA) studies were done shortly after the onset of the symptoms. The AICA vascular territory was identified using the Amarenco and Hauw anatomical diagrams ${ }^{3}$ (involving at least one of the following anatomical structures: middle cerebellar peduncle, lateral inferior pontine area, anterior cerebellar hemisphere).

\section{RESULTS}

Among 16 consecutives patients with AICA infarction, we identified five (three women and two men, aged 60 to 71 years) who had an isolated acute auditory syndrome, such as hearing loss or tinnitus, as a prodromal feature. This occurred one to 10 days before the onset of the definite brain stem or cerebellar infarction. The clinical data are summarised in table 1. All patients had at least one vascular risk factor for cerebrovascular disease. Three patients (Nos 1, 2, and 5) had recurrent episodes of transient hearing loss with or without tinnitus unilaterally. One patient (No 3) had a single prolonged episode of tinnitus and hearing loss on the both sides and another (No 4) had a single prolonged episode of hearing loss unilaterally. Six, including the five patients described here, also had episodes of transient vertigo before the AICA infarction. The patients reported that the prodromal tinnitus and vertigo were identical to the symptoms that occurred during the actual pontocerebellar AICA infarct. These episodes of acute auditory syndrome and vertigo were brief, lasting minutes.

At the time of the infarction, all patients developed hearing loss, vertigo, and hemiataxia. T2 weighted MRI of the brain showed hyperintense lesions in the middle cerebellar 
Table 1 Baseline clinical profile of five patients with an acute auditory syndrome as a prodromal feature of impending AICA infarction

\begin{tabular}{|c|c|c|c|c|c|}
\hline $\begin{array}{l}\text { Patient } \\
\text { No }\end{array}$ & Age/sex & $\begin{array}{l}\text { Vascular risk } \\
\text { factors }\end{array}$ & AAS (prodromal symptoms) & MRI & MRA \\
\hline $1^{*}$ & $62 / \mathrm{F}$ & HTN & $\begin{array}{l}\text { Tinnitus and hearing loss on } R \\
\text { (10 days) }\end{array}$ & MCP DLP & Proximal BA stenosis \\
\hline $2^{*}$ & $71 / \mathrm{F}$ & HTN & Tinnitus on $\mathrm{R}$ (1 days) & MCP AICH & Diffuse BA stenosis \\
\hline $3+$ & $67 / M$ & NIDDM & $\begin{array}{l}\text { Hearing loss on } B \text { and tinnitus on } R \\
(10 \text { days) }\end{array}$ & $\begin{array}{l}\text { MCP DLP } \\
\text { AICH }\end{array}$ & Mid BA stenosis \\
\hline $4 \dagger$ & $60 / \mathrm{F}$ & NIDDM, HTN & Hearing loss on L ( 6 days) & MCP DLP & Mid BA stenosis \\
\hline $5^{\star}$ & $62 / M$ & NIDDM, HTN & Hearing loss on $R$ and tinnitus ( 4 days) & MCP DLP & Proximal BA stenosis \\
\hline
\end{tabular}

MRI and MRA were done at the time of anterior inferior cerebellar artery infarction.

*Patients had recurrent episodes of transient acute auditory syndrome before infarction.

†Patients had a single episode of prolonged acute auditory syndrome before infarction.

AAS, acute auditory symptom; AICH, anterior inferior cerebellar hemisphere; B, both; BA, basilar artery; DLP,

dorsolateral pons; $F$, female; HTN, hypertension; L, left; M, male; MCP, middle cerebellar peduncle; MRA, magnetic resonance angiography; MRI, magnetic resonance imaging; NIDDM, non-insulin-dependent diabetes mellitus; $R$, right.

peduncle $(n=5)$, dorsolateral pons $(n=4)$, and anterior inferior cerebellar hemisphere $(n=2)$. Four patients (Nos 1 , 3,4 , and 5) had a focal stenosis near the origin of the AICA and the remaining patient (No 2) had diffuse basilar occlusive disease on MRA. At the time of infarction, pure tone audiometry confirmed sensorineural hearing loss in all five patients, unilateral in four (Nos 1, 2, 3, and 5) and bilateral in one (No 4). Hearing impairment was mild in two patients (Nos 1 and 5), moderate in two (Nos 3 and 4), and severe in one (No 2). None of the patients had a previous history of expose to a noisy environment, head trauma, meningitis, ototoxic drugs, or syphilis. All patients with hearing loss noted a definite change in hearing along with their other neurological symptoms at the time of infarction. Furthermore, in all patients except one (No 3), the unilateral hearing loss was on the side of infarct, as shown on brain MRI. From the findings of the pure tone audiometry, stapedial reflex testing, and ABR, the hearing loss was localised to the cochlea in four patients (Nos 1, 3, 4, and 5). ABR showed normal waveform responses in two patients (Nos 3 and 4) with a moderate hearing loss on pure tone audiometry, and in two patients (Nos 1 and 5) with mild hearing loss. The remaining patient (No 2) had severe hearing loss on pure tone audiometry and no wave responses on ABR testing. Electronystagmography showed no response to caloric stimulation in the affected ear in any of the five patients.

\section{DESCRIPTION THE CASES}

Patient 1: episodic prodromal unilateral hearing loss and tinnitus

A 62 year old woman with hypertension developed three episodes of transient left sided tinnitus and hearing loss lasting a few minutes 10 days before admission. Nine days later, she had two episodes of transient vertigo lasting several minutes without any accompanying auditory or neurological symptoms. A day after onset of the vertigo, she suddenly developed vertigo, nausea, vomiting, left sided tinnitus, and unsteadiness. On admission, she had a gaze evoked bidirectional horizontal nystagmus, hypalgesia over the left half of the face, left limb dysmetria and facial weakness, and gait ataxia. MRI of the brain showed hyperintense lesions on axial T2 weighted images situated in the left middle cerebellar peduncle and the left lateral pons. Pure tone audiometry showed a mild $(40 \mathrm{~dB})$ sensorineural hearing loss on the left side. Stapedial reflexes were recorded at normal levels from both sides. Normal waveform responses were evoked bilaterally on ABR testing. Electronystagmography showed no response to caloric stimulation of the left side. During several days of anticoagulation in the hospital, the patient's vertigo and nausea improved.

\section{Patient 2: episodic prodromal unilateral tinnitus}

A 60 year old woman with long standing hypertension was admitted to our hospital because of the sudden onset of vertigo, right sided hearing loss and tinnitus, and vomiting. Two months before admission, she had had two episodes of transient vertigo lasting a few minutes without any accompanying neurological symptoms. One day before admission, she complained of sudden onset of transient vertigo and right sided tinnitus. Describing the tinnitus, she noted, "the wind sounded like an airplane." The vertigo and tinnitus spontaneously subsided within several minutes. On admission, she had gaze evoked bidirectional horizontal nystagmus, right limb dysmetria and facial weakness, and gait ataxia. MRI of the brain showed hyperintense lesions on axial T2 weighted images situated in the right middle cerebellar peduncle and the right anterior cerebellum. Pure tone audiometry showed a severe $(80 \mathrm{~dB})$ sensorineural hearing loss on the right side. On electronystagmography there was no response to caloric stimulation on the right side. Right limb coordination and gait improved steadily over several weeks. Follow up pure tone audiometry at six months showed that the hearing loss in the right side had improved to $35 \mathrm{~dB}$.

\section{Patient 3: prolonged bilateral hearing loss and tinnitus as prodrome}

A 67 year old man with type II diabetes mellitus had acute onset of bilateral hearing loss and vertigo, preceded by right sided tinnitus on awakening. The patient described the tinnitus as a sudden ringing sound in the right ear. The hearing loss persisted, but the vertigo and tinnitus improved within a day. He came to our hospital because of exacerbation of right sided hearing loss and vertigo 10 days later. The tinnitus (a ringing sound) at the time of admission was identical to that experienced preceding admission. Examination revealed a gaze evoked bidirectional horizontal nystagmus with a torsional component. There was diminished right sided facial sensation, a right sided peripheral facial palsy, and limb dysmetria on the right side. Pure tone audiometry showed a moderate sensorineural hearing loss on the both sides. On ABR testing, normal wave responses were evoked by stimulation on the both sides. Stapedial reflexes were also recorded at normal levels from both sides. Electronystagmography showed no response to caloric stimulation in the right ear. T2 weighted MRI revealed hyperintense foci involving the right dorsolateral pons, right middle cerebellar peduncle, and the right anterior inferior 


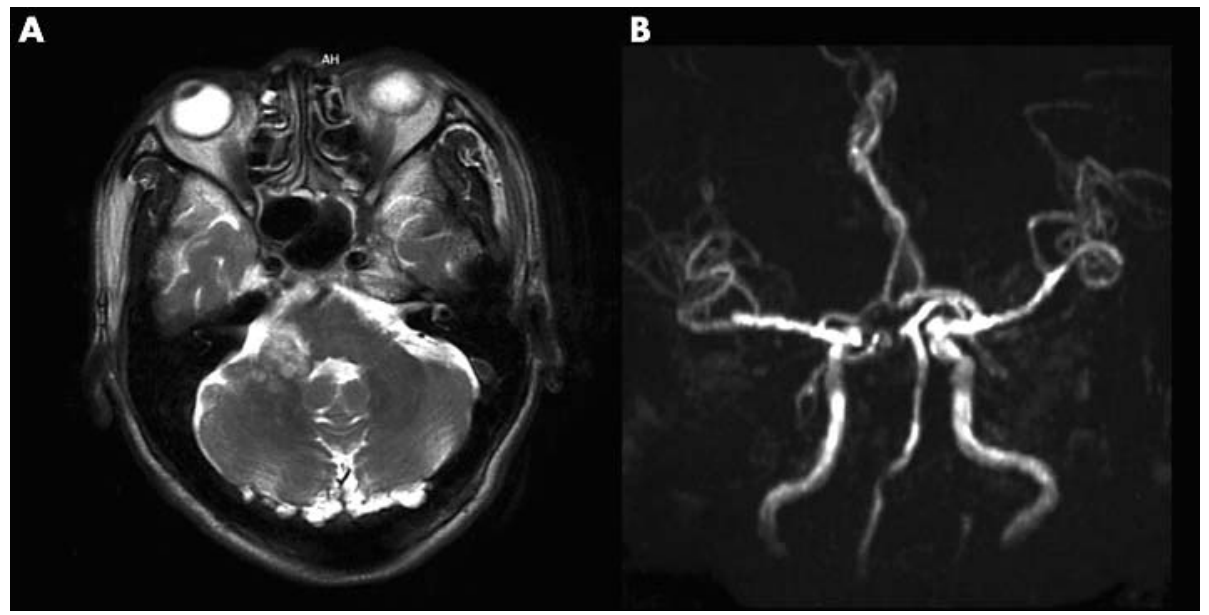

Figure 1 Magnetic resonance imaging findings in patient 3, with prolonged bilateral hearing loss and tinnitus as the prodrome. (A) T2 weighted axial image of the brain showing hyperintense lesions involving the right dorsolateral pons, right middle cerebellar peduncle, and the right anterior cerebellar hemisphere. (B) Magnetic resonance angiography showings moderate stenosis of the mid-basilar artery and distal right vertebral artery.

cerebellar hemisphere. MRA showed moderately severe stenosis of the distal right vertebral artery and the middle third of the basilar artery (fig 1). Following a three week hospital admission, a follow up audiogram showed profound hearing loss on the right, but the hearing loss on the left had improved to $30 \mathrm{~dB}$ (fig 2). On ABR testing, no responses were evoked by stimulation on the right side, whereas there were normal waveforms on the left. No stapedial reflexes were elicited from the right side. Right limb coordination and gait improved steadily over several weeks, but right sided hearing loss remained.

\section{Patient 4: prolonged unilateral hearing loss as the prodrome}

A 60 year old woman with type 2 diabetes mellitus and hypertension was admitted to our hospital because of the sudden onset of vertigo, vomiting, and hearing loss on the left side. Three days before admission, she had two episodes of transient vertigo lasted 10 minutes without any accompanying neurological symptoms. On examination, she had a unidirectional right beating nystagmus with a horizontorotatory component on primary positioning and on gazing to both sides. Pure tone audiometry showed a moderate sensorineural hearing loss of $50 \mathrm{~dB}$ on the left side. Stapedial reflexes were recorded at normal levels from both sides. Normal waveform responses were evoked bilaterally on ABR testing. Electronystagmography showed no response to caloric stimulation on the left side. MRI revealed that the middle cerebellar peduncle and dorsolateral pons were spared. Low dose aspirin (100 mg) treatment was started. The hearing loss persisted, but the vertigo improved steadily over a few days. Six days after the initial onset of vertigo and hearing loss in the left side, the patient complained of an exacerbation of the vertigo, combined with nausea, vomiting, and imbalance. On neurological examination, she had a gaze evoked bidirectional horizontal nystagmus. There was diminished left sided facial sensation and dysmetria of the left limbs. Follow up MRI showed new infarcts in the left middle cerebellar peduncle and the left dorsolateral pons. Over several days of hospital admission, the vertigo, nausea, and vomiting subsided. Follow up pure tone audiometry showed the hearing loss in the left side had improved to $35 \mathrm{~dB}$.

\section{Patient 5: episodic prodromal unilateral hearing loss and tinnitus}

A 62 year old man with type II diabetes mellitus and hypertension developed two episodes of transient right sided hearing loss and tinnitus four days before admission. He described the tinnitus as a sudden buzzing sound in the right ear. The tinnitus and hearing loss occurred while sitting in the chair, lasted 10 minutes, and were not accompanied by any neurological symptoms except mild unsteadiness on walking. Three days later, he had three episodes of transient vertigo lasting several minutes without any accompanying
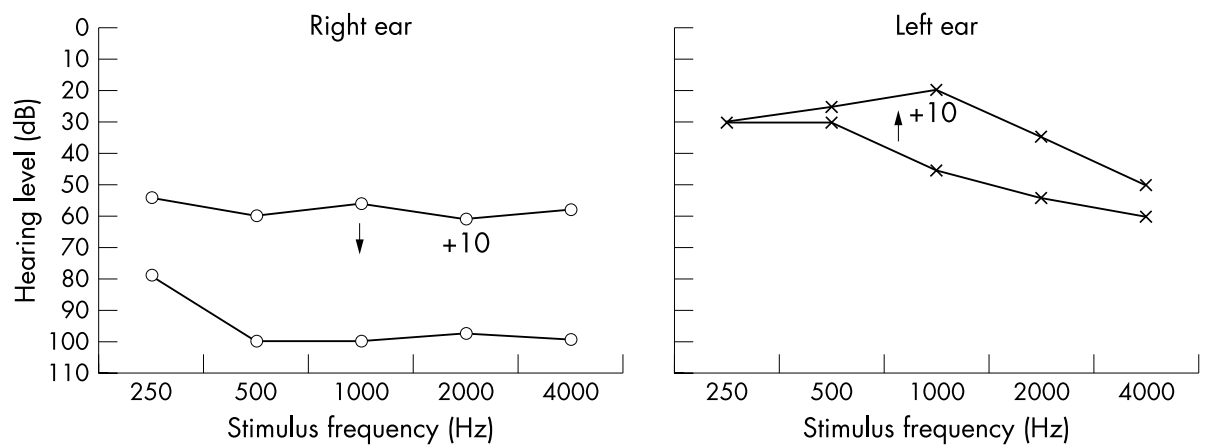

Figure 2 Audiological findings in patient 3 with prolonged bilateral hearing loss and tinnitus as the prodrome. Pure tone air conduction audiograms obtained on two different dates, 10 days apart $(+10)$. The patient's hearing levels are plotted against stimulus frequency on a logarithmic scale. The measurements were made according to the American National Standards Institute, 1989. Circles, air conduction on the right side; crosses, air conduction on the left side. 
auditory or neurological symptoms. The next day he had a recurrence of vertigo accompanied by right sided hearing loss and tinnitus, vomiting, and severe ataxia. The tinnitus (a buzzing sound) was identical to that experienced preceding his admission.

On neurological examination, he had a gaze evoked bidirectional horizontal nystagmus, hypalgesia over the right half of the face, right limb dysmetria, facial weakness, and gait ataxia. Axial T2 weighted brain MRI showed hyperintense lesions situated in the right middle cerebellar peduncle and right dorsolateral pons. MRA showed proximal basilar stenosis close to the origin of the AICA (fig 3). Pure tone audiometry revealed a mild $(37 \mathrm{~dB})$ sensorineural hearing loss on the right side. (fig 4). Stapedial reflexes were recorded at normal levels from both sides. Normal waveform responses were evoked bilaterally on ABR testing. Electronystagmography showed no response to caloric stimulation of the right ear. The vertigo and nausea resolved but hearing loss persisted at a four months follow up.

\section{DISCUSSION}

In all five of patients, an episodic or prolonged acute auditory syndrome preceded other brain stem or cerebellar signs by a period of one to 10 days. Two patterns of basilar occlusive disease were seen: focal stenosis near the origin of the AICA, and diffuse basilar stenosis. In episodic acute auditory syndrome, hearing loss and tinnitus were remarkably similar-an abrupt onset lasting a few minutes and rapid disappearance. All patients with acute auditory syndrome also had transient vertigo lasting a few minutes, consistent with a transient ischaemic attack in the vertebrobasilar circulation. ${ }^{9}$ Furthermore, the tinnitus preceding the infarction was identical to that experienced at the time of infarction, and isolated vertigo preceding the infarction was identical in quality to the vertigo experienced at the time of infarction.

Most previous reports of an isolated neuro-otological symptom as a manifestation of vertebrobasilar insufficiency have focused on the acute vestibular symptom of vertigo. ${ }^{10-17}$ There have been few reports of an acute auditory syndrome as an initial manifestation of vertebrobasilar insufficiency. In 1981, Stephan et al described a patient with sudden bilateral hearing loss caused by basilar artery occlusion who later developed multiple brain stem dysfunctions with quadriplegia and mental change. ${ }^{6}$ In 1993, Huang et al described seven patients with sudden bilateral hearing loss caused by vertebrobasilar occlusive disease. ${ }^{7}$ Six had an acute auditory

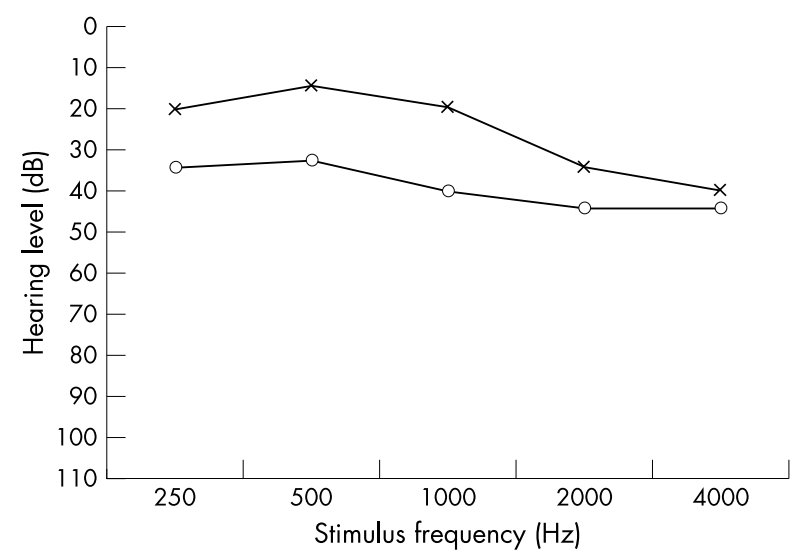

Figure 4 Pure tone air conduction audiograms in patient 5 with episodic prodromal unilateral hearing loss and tinnitus. The patient's hearing levels are plotted against stimulus frequency on a logarithmic scale. Measurements were made according to the American National Standards Institute, 1989. Circles, air conduction on the right side; crosses, air conduction on the left side.

syndrome as the initial presenting symptom but three also had focal neurological signs at onset, such as quadriparesis, hemianaesthesia, dysarthria, and facial palsy. The report did not include audiometric data, which are essential for localising the site of the sudden hearing loss. In 1998, Deplanque et al reported a patient with unilateral lateral inferior pontine infarction who presented with bilateral deafness, facial palsy, Horner's syndrome, and ataxia. ${ }^{18}$ In 2002, Toyoda et al reported two patients with basilar artery occlusion who had bilateral hearing loss as a warning sign of an impending stroke. ${ }^{19}$ No large consecutive clinical series of AICA infarction has focused on an acute auditory syndrome as a warning sign of impending infarction.

It is well recognised that the tinnitus is a very common complaint, not only in association with deafness but also in individuals with normal hearing, and it is not usually taken as a warning sign of an impending stroke. However, during the episodes of tinnitus, the patient No 2 also complained of episodic vertigo lasting a few minutes, which is a typical duration of ischaemia within the posterior circulation.' Furthermore, the quality of tinnitus was identical to the tinnitus experienced at the time of infarction.

It was surprising that all the patients had total canal paresis to caloric stimulation yet an incomplete hearing loss

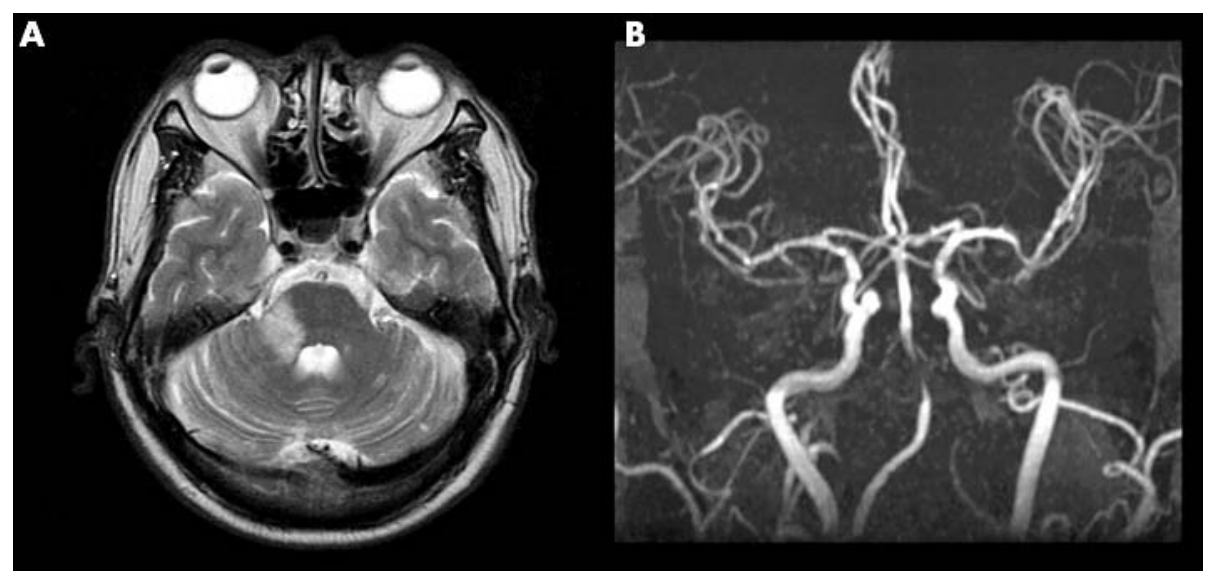

Figure 3 Magnetic resonance imaging findings in patient 5 with episodic prodromal unilateral hearing loss and tinnitus. (A) T2 weighted axial image of the brain shows a hyperintense lesion situated in the right middle cerebellar peduncle and right dorsolateral pons. (B) Magnetic resonance angiography shows proximal basilar artery stenosis close to the origin of the anterior inferior cerebellar artery. 
ipsilaterally. However, the absence of caloric responses does not indicate absence of vestibular function. ${ }^{20}$ Most of the patients in our AICA infarct group had normal responses to rotational testing at higher frequencies (up to $0.8 \mathrm{~Hz}$ ).

Without pathological confirmation, it is difficult to localise precisely the lesion responsible for prolonged acute auditory syndrome with hearing loss in two patients (patients 3 and 4). However, stapedial reflex testing and auditory brain stem responses were normal, implicating the cochlea as the site of injury. ${ }^{21} 22$ A cochlear site for hearing loss is not surprising owing to its high metabolic energy requirement and demonstrated lack of collateral circulation. ${ }^{1811}$ By contrast, the retrocochlear acoustic nerve has an abundant collateral blood supply arising from the lateral medullary artery, the arteries supplying adjacent dura matter and petrous bone, and the inferior lateral pontine artery. ${ }^{23}{ }^{24}$ In future studies, measurement of otoacoustic emissions might help to localise the site of hearing loss better.

It is interesting to compare our MRA findings in the patients with AICA infarction and an acute auditory syndrome with those in patients without that feature: focal stenosis of the basilar artery close to the origin of the AICA on brain MRA was more common in the patients with acute auditory syndrome $(80 \% v 18.2 \%, \mathrm{p}<0.01)$. This finding may explain the high incidence of prodromal symptoms in the group of patients with an acute auditory syndrome.

We were surprised to find a relatively high incidence of acute auditory syndrome as a warning sign of an impending AICA stroke. While vascular studies of the vestibular labyrinth have shown that the superior labyrinth is more susceptible to ischaemia than the inferior, ${ }^{11}$ experimental studies of transient internal auditory artery occlusion have revealed that the cochlea is more sensitive to ischaemia than the vestibular labyrinth. ${ }^{25}{ }^{26}$ Previous reports have suggested that an isolated acute auditory syndrome is less common than vertigo in vertebrobasilar insufficiency. ${ }^{7} 1627$ This is in part because the broad projection of the vestibular pathways is highly likely to result in symptoms, while hearing loss results from ischaemia restricted to the peripheral cochlear system. Moreover, patients are more sensitive to a disturbance of the vestibular system than to diminished hearing, especially if one side is affected. ${ }^{1828}$ All these factors may explain the relatively low incidence of acute auditory syndrome in previous reports of vertebrobasilar insufficiency. We speculated that a prominence of an acute auditory syndrome and the absence of other neurological symptoms or signs is best explained by the selectively vulnerability of the cochlea to ischaemia.

\section{Conclusions}

Episodic or prolonged acute auditory syndrome without neurological symptoms or signs can be the initial manifestation of AICA infarction. Clinicians should be aware that an isolated acute auditory syndrome may herald an impending AICA infarct.

\section{ACKNOWLEDGEMENT}

This work was supported by a research promoting grant from the Keimyung University Dongsan Medical Centre.

\section{Authors' affiliations}

H Lee, Y-W Cho, Department of Neurology, School of Medicine and Institute for Medical Science, Keimyung University School of Medicine, Daegu, South Korea

Competing interests: none declared

\section{REFERENCES}

1 Oas JG, Baloh RW. Vertigo and the anterior inferior cerebellar artery syndrome. Neurology 1992;42:2274-9.

2 Adams RD. Occlusion of the anterior inferior cerebellar artery. Arch Neurol Psychiatry 1943;49:765-70.

3 Amarenco $P$, Hauw JJ. Cerebellar infarction in the territory of the anterior and inferior cerebellar artery. Brain 1990;113:139-55.

4 Milander L, Rumeau C, Sangla I, et al. Infarction in the territory of the anterior inferior cerebellar artery: reports of five cases. Neuroradiology 1992;34:500-3.

5 Amarenco P, Rosengart A, Dewitt LD, et al. Anterior inferior cerebellar artery territory infarcts. Mechanism and clinical features. Arch Neurol 1993;50:154-61.

6 Stephan H, Wappenschmidt J, Kiefer H. Occlusion of the basilar artery. Neurosurg Rev 1981;4:41-52.

7 Huang MH, Huang CC, Ryu SJ, et al. Sudden bilateral hearing impairment in vertebrobasilar occlusive disease. Stroke 1993;24:132-7.

8 Lee H, Sohn II, Jung DK, et al. Sudden deafness and anterior inferior cerebellar artery infarction. Stroke 2002;33:2807-12.

9 Kimura K, Minematsu K, Yasaka M, et al. The duration of symptoms in transient ischemic attack. Neurology 1999;52:976-80.

10 Pessin MS, Gorelick PB, Kwan ES, et al. Basilar artery stenosis: middle and distal segments. Neurology 1987;37:1742-6.

11 Grad A, Baloh RW. Vertigo of vascular origin: clinical and electronystagmographic features in 84 cases. Arch Neurol 1989;46:281-4.

12 Ferbert A, Bruckmann H, Drummen R. Clinical features of proved basilar artery occlusion. Stroke 1990;21:1135-42.

13 Fife TD, Baloh RW, Duckwiler GR. Isolated dizziness in vertebrobasilar insufficiency: clinical features, angiography, and follow-up. J Stroke Cerebrovasc Dis 1994:4:4-12.

14 Hemanth Rao T, Libman RB. When is isolated vertigo a harbinger of stroke. Ear Nose Throat J 1995;74:33-6.

15 Norrving B, Magnusson M, Holtas S. Isolated acute vertigo in the elderly; vestibular or vascular disease? Acta Neurol Scand 1995;91:43-8.

16 Gomez CR, Cruz-Flores S, Malkoff MD, et al. Isolated vertigo as a manifestation of vertebrobasilar ischemia. Neurology 1996;47:94-7.

17 Berlit P. Isolated vertigo in vertebrobasilar ischemia-does it exit? HNO 1998:46:296-300.

18 Deplanque D, Godeferoy D, Guerouaou D, et al. Sudden bilateral deafness: lateral inferior pontine infarction. J Neurol Neurosurg Psychiatry 1998;64:817-18.

19 Toyoda K, Hirano T, Kumai Y, et al. Bilateral deafness as a prodromal symptom of basilar artery occlusion. J Neurol Sci 2002; 193:147-50.

20 Baloh RW, Honrubia V, Yee RD, et al. Changes in the human vestibulo-ocular reflex after loss of peripheral sensitivity. Ann Neurol 1984; 16:222-8.

21 Baloh RW, Honrubia V. Clinical neurophysiology of the vestibular system, 3rd ed. New York: Oxford University Press, 2001:200-14.

22 Baloh RW. Dizziness, hearing loss and tinnitus. New York: Oxford University Press, 1988:89-105.

23 Mazzoni A. Internal auditory canal arterial relations at the porus acusticus. Ann Otol Rhinol Laryngol 1969;78:797-814.

24 Mazzoni A. Internal auditory artery supply to the petrous bone. Ann Otol Rhinol Laryngol 1972;81:13-21.

25 Perman HB, Kimura RS, Fernandez C. Experiments on temporal obstruction of the internal auditory artery. Laryngscope 1959;69:591-613.

26 Levine RA, Bu-Saba N, Brown MC. Laser-Doppler measurements and electrocochleography during ischemia of the guinea pig cochlea: implication for hearing preservation acoustic neuroma surgery. Ann Otol Rhinol Laryngol 1993; 102:127-36.

27 Ymasoba T, Kikuchi S, Higo R. Deafness associated with vertebrobasilar insufficiency. J Neurol Sci 2001;187:69-75.

28 Halmahyi GM, Cremer PD. Assessment and treatment of dizziness. J Neurol Neurosurg Psychiatry 2000;68:129-34. 